\title{
Epstein-Barr Virus-Associated Lymphoproliferative Disorders: Review and Update on 2016 WHO Classification
}

Hyun-Jung Kim • Young Hyeh Ko ${ }^{1}$ Ji Eun Kim² · Seung-Sook Lee ${ }^{3}$ Hyekyung Lee ${ }^{4}$. Gyeongsin Park ${ }^{5}$ Jin Ho Paik ${ }^{6} \cdot$ Hee Jeong Cha ${ }^{7}$ Yoo-Duk Choi ${ }^{8} \cdot$ Jae Ho Han ${ }^{9}$ Jooryung Huh ${ }^{10} \cdot$ Hematopathology Study Group of the Korean Society of Pathologists

Department of Pathology, Inje University, Sanggye Paik Hospital, Seoul; ' ${ }^{S}$ Sungkyunkwan University, School of Medicine, Samsung Medical Center, Seoul; ' 2 SMG-SNU Boramae Medical Center, Seoul National University, Seoul; ${ }^{3}$ Korea Cancer Center Hospital, Seoul; ${ }^{4}$ Eulji University Hospital, Eulji University School of Medicine, Daejeon; ${ }^{5}$ Gangnam St. Mary's Hospital, College of Medicine, The Catholic University of Korea, Seoul; ${ }^{6}$ Seoul National University Bundang Hospital, Seongnam; 'Ulsan University Hospital, Ulsan University School of Medicine, Ulsan; ${ }^{8}$ Chonnam National University Hospital, Chonnam National University, Gwangju; ${ }^{9}$ Ajou University Hospital, Suwon; ${ }^{10}$ Asan Medical Center, Ulsan University College of Medicine, Seoul, Korea

Received: January 6, 2017

Revised: March 11, 2017

Accepted: March 14, 2017

Corresponding Author

Jooryung Huh, MD

Department of Pathology, Asan Medical Center,

University of Ulsan College of Medicine, 88

Olympic-ro 43-gil, Songpa-gu, Seoul 05505, Korea

Tel: +82-2-3010-4553

Fax: +82-2-472-7898

E-mail: Jrhuh@amc.seoul.kr
Epstein-Barr virus (human herpesvirus-4) is very common virus that can be detected in more than $95 \%$ of the human population. Most people are asymptomatic and live their entire lives in a chronically infected state (IgG positive). However, in some populations, the Epstein-Barr virus (EBV) has been involved in the occurrence of a wide range of B-cell lymphoproliferative disorders (LPDs), including Burkitt lymphoma, classic Hodgkin's lymphoma, and immune-deficiency associated LPDs (post-transplant and human immunodeficiency virus-associated LPDs). T-cell LPDs have been reported to be associated with EBV with a subset of peripheral T-cell lymphomas, angioimmunoblastic T-cell lymphomas, extranodal nasal natural killer/T-cell lymphomas, and other rare histotypes. This article reviews the current evidence covering EBV-associated LPDs based on the 2016 classification of the World Health Organization. These LPD entities often pose diagnostic challenges, both clinically and pathologically, so it is important to understand their unique pathophysiology for correct diagnoses and optimal management.

Key Words: Epstein-Barr virus; Lymphoproliferative disorders
Epstein-Barr virus (EBV) is classified as a $\gamma$-herpes virus and contains a linear DNA molecule about $172 \mathrm{~kb}$ in length, which affects more than $90 \%$ of the worldwide adult population. If the infection does not become clinically silent, infectious mononucleosis is experienced by the exposed persons. ${ }^{1}$ Although EBV infection is lifelong, a long latency and reactivation of EBV results in various lymphoproliferative lesions including hematologic malignancies. ${ }^{2}$ This article reviews the current understanding of EBV-associated lymphoproliferative disorders (LPDs) based on the 2016 classification of the World Health Organization 
(WHO). ${ }^{3}$ Some LPDs exhibit a predisposition in Asian populations, including Koreans. These entities often pose diagnostic challenges, both clinically and pathologically, and it is important to understand their unique pathophysiology for correct diagnoses and optimal management. Generally intrinsic defects and post-transplant lymphoproliferative disorders (PTLDs) are excluded from this review, these are treated separately as immunodeficiency disorders by the WHO.

\section{EPSTEIN-BARR VIRUS-ASSOCIATED B-CELL LYMPHOPROLIFERATIVE DISORDERS}

The spectrum of EBV-associated B-cell LPDs is broad, ranging from reactive lymphoproliferative lymphadenitis to lymphomas. All the related disease entities are shown in Table 1. Welldefined lymphoma entities such as Burkitt lymphoma, classical Hodgkin lymphoma, and plasmablastic lymphoma are not included. $^{4}$

\section{Infectious mononucleosis}

Primary EBV infection occurs most often in childhood and is generally asymptomatic. In adolescence, it is associated with a self-limiting infectious mononucleosis syndrome, manifested by fever, pharyngitis, malaise, and atypical lymphocytosis. Following primary infection, most individuals remain a life-long carrier of the virus without serious sequelae. ${ }^{5}$ However, a small population with the latent infection will develop various LPDs.

EBV is transmitted from the host by saliva and infected EBV replicates within oropharyngeal epithelium and is then exposed to circulating B-lymphocytes. Peripheral EBV-infected memory B-cells can return to the Waldeyer's ring, undergoing reactivation to produce an infectious virus that will be shed in the saliva. EBV-specific cytotoxic T-cells (CTL) destroy most infected cells.

The histologic features of infectious mononucleosis vary during the course of the disease. Early in the disorder, follicular hyperplasia occurs with monocytoid B-cell aggregates and epithelioid histiocytes. Later, the expansion of the paracortex predominates. The immunoblasts resemble classical Reed-Sternberg (RS) cells. The immunoblasts of predominantly B-cell types and partly T-cell types often express CD30. In-situ hybridization (ISH) of EBVencoded small RNAs (EBERs) exhibit numerous positive immunoblasts in the paracortex but not in the germinal centers. ${ }^{6}$

\section{Chronic active EBV of B-cell types}

As first defined by Lekstrom-Himes et al., chronic active EBV (CAEBV) of B-cell types refers to a chronic or persistent EBV in- fection characterized by a severe illness lasting more than 6 months, persistent elevated EBV titers, and evidence of EBV-related organ damage. Currently, CAEBV is defined as (1) a severe progressive illness with a duration of more than 6 months, (2) lymphocytic infiltration of tissue (e.g., lymph nodes, lungs, liver, central nervous tissue, bone marrow, eye, and skin), (3) elevated EBV DNA and RNA in affected tissue, and (4) absence of any other immunosuppressive conditions. ${ }^{8}$

Histologically, the lymph nodes exhibits features resembling polymorphic PTLD, with paracortical expansion, plasmacytoid lymphoblastic proliferation, presence of plasma cells, and presence of occasional RS-like cells. EBV-ISH positive B-cells are noted in the paracortex. Among CAEBV patients, $63 \%$ had clonal immunoglobulin rearrangement. ${ }^{\text {? }}$

\section{EBV-positive diffuse large B-cell lymphoma}

EBV-positive diffuse large B-cell lymphoma (DLBCL), not otherwise specified was originally described as "senile EBV-associated B-cell LPD," or "EBV-positive DLBCL of the elderly" (older than 50 years) (WHO 4th edition). ${ }^{10}$ However, subsequent studies have shown that EBV-positive DLBCL is not limited to this older age group. In the elderly group, it is thought to be related to immunosenescence, which modifies T-cell homeostasis through a lack of thymic output of naïve T-cells and an accumulation of viral specific $\mathrm{CD} 8^{+} \mathrm{T}$ cells.

Histologically, four types have been described: monomorphic (DLBCL-like, monotonous sheets of large cells), polymorphic in the inflammatory background, T-cell/histiocyte-rich large cell lymphoma, and plasmacytoid differentiation. Immunophenotypically, the tumor cells express pan B-cell markers (CD20, PAX5, CD79a, OCT-2, and BOB-1), and are mostly $\mathrm{CD} 30^{+}$, but lack CD15 expression.

This disease entity frequently involves extranodal sites including the skin, lung, tonsils, and stomach. Unfavorable prognostic factors include older age ( $>70$ years), high international prognostic index, and activated B-cell phenotype. ${ }^{11}$

\section{EBV mucocutaneous ulcer}

This self-limited EBV positive B-cell proliferation is characterized by the presence of mucocutaneous ulcers with an indolent clinical course. This phenomenon is likely due to a more localized form of decreased immune surveillance, which is supported by a very low EBV viral load. The frequently involved sites includes the skin, oropharyngeal mucosa, and gastrointestinal tract. ${ }^{12}$

Histologically a sharply demarcated ulcer is lined by an inflammatory infiltrate with clusters of large atypical cells, often 
with RS cell-like features. Phenotypically the large atypical cells are variably positive for $\mathrm{CD} 20$ and $\mathrm{CD} 30$, and uniformly positive for $\mathrm{EBV}-\mathrm{ISH}$ and $\mathrm{CD} 15^{+}$in half of the cases (Fig. 1).

\section{DLBCL associated with chronic inflammation}

DLBCL associated with chronic inflammation develops in the setting of long-standing chronic inflammation with EBV association. It usually involves body cavities or enclosed spaces (like cysts). Pyothorax-associated lymphoma (PAL) represents the prototype of this entity.

Cases with PAL have long history of chronic pyothorax and may present with chest pain, fever, cough, dyspnea, and tumor mass. The prognosis of PAL is poor.

The morphology discloses a diffuse proliferation of large atypical lymphocytes with plasmacytoid cytomorphology. Immunohistochemistry reveals neoplastic cells that represent pan-B cell markers, usually positive for IRF4/MUM1, and CD13. An aberrant expression of T-cell phenotypes is also seen.

The unique genomic instability has been reported as follows: A20 deletion, interferon-inducible 27 (IFI27), TP53 mutation, and MYC amplification. ${ }^{13}$

\section{Lymphomatoid granulomatosis}

Katzenstein et al.$^{14}$ initially described a rare angiocentric and angiodestructive EBV-associated LPD, which was distinct from Wegener's granulomatosis.

Nearly all patients present with symptoms related to pulmonary involvement, followed by involved sites of the central nervous system, skin, liver, and kidney. Radiologically, bilateral variable sized lung nodules are noted in lymphomatoid granulomatosis (LYG).

The histologic features of LYG are observed in lung nodules. All the lesions are angioinvasive and angiocentric with fibrinoid necrosis of the vascular wall. The infiltrate is polymorphous with an admixture of small lymphocytes, histiocytes, and large lymphoid cells. The grading of LYG is based on the proportion of

Table 1. Epstein-Barr virus-associated B-cell lymphoproliferative diseases

\begin{tabular}{l}
\hline Disease \\
\hline Infectious mononucleosis \\
Chronic active Epstein-Barr virus of B-cell type \\
Epstein-Barr virus-positive diffuse large B-cell lymphoma \\
Epstein-Barr virus mucocutaneous ulcer \\
Diffuse large B-cell lymphoma associated with chronic inflammation \\
Lymphomatoid granulomatosis
\end{tabular}
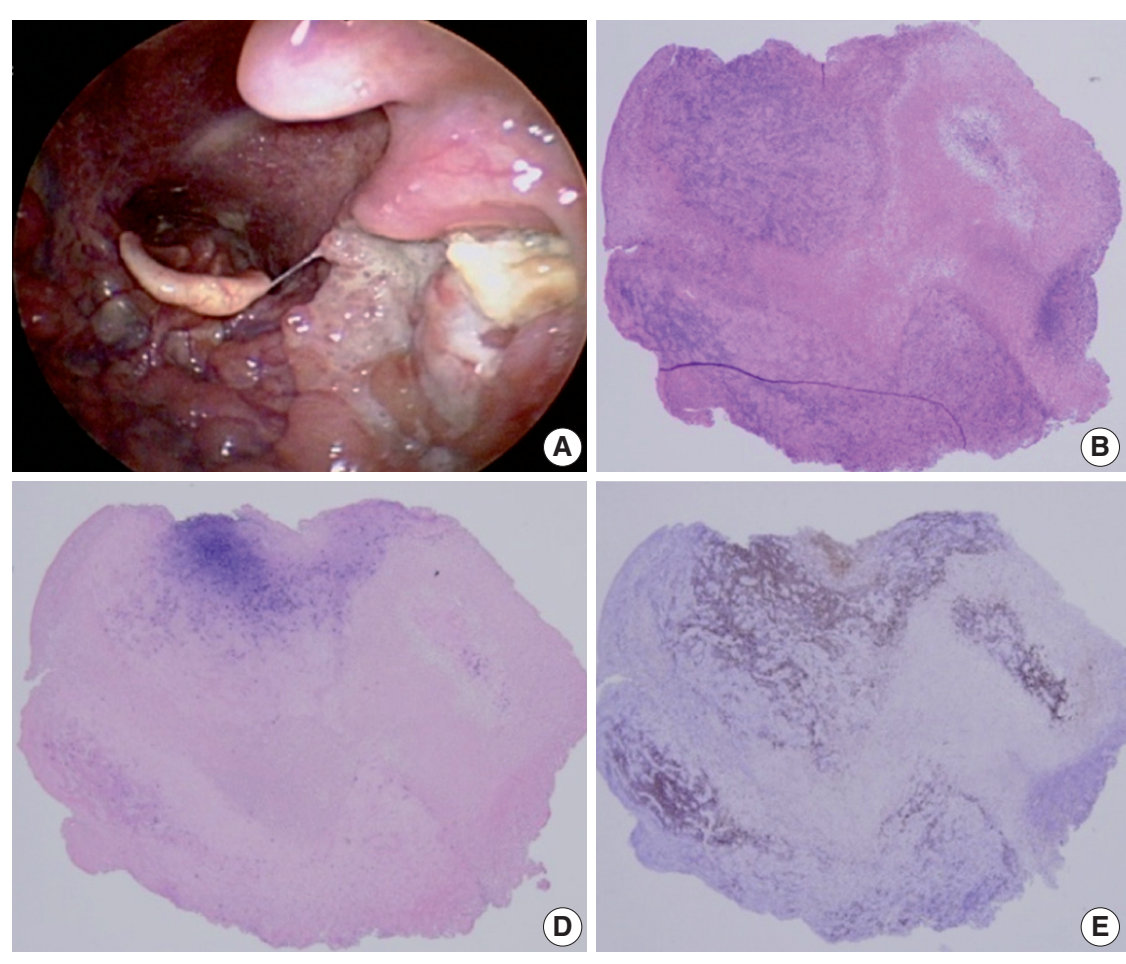

(E)

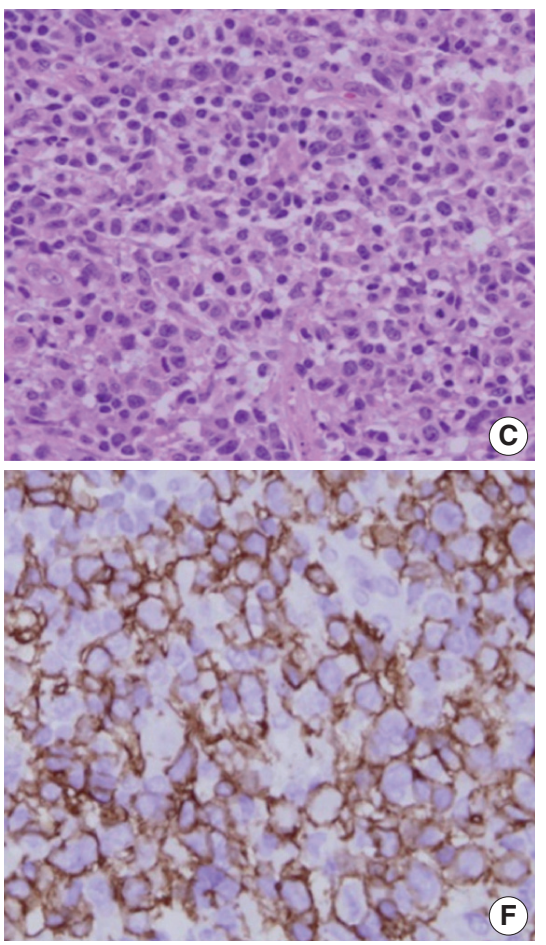

Fig. 1. Mucocutaneous ulcer (Courtesy of Dr. J.H. Paik). (A) This 70-year-old female presented with a sore throat, painful swelling saliva, and tonsillar enlargement with a discrete ulcer. (B) The scanning power view shows a dense infiltrate beneath the ulcer. (C) Medium sized atypical lymphocytes are observed. (D) Epstein-Barr virus (EBV)-in-situ hybridization positive cells are aggregated in the ulcer bed. (E) CD20 immunostaining disclosed overlapping with EBV-positive cells. (F) The large atypical cells are diffusely and strongly positive for CD20. 
EBV positive cells. In general, grade 1 and grade 2 lesions are approached using strategies that are designed to improve the host's immune system, whereas grade 3 lesions require chemotherapy and do not respond to immunomodulatory therapies. Phenotypically, large atypical EBV-positive B cells express CD20, PAX5, $\mathrm{CD} 79 \mathrm{a}, \mathrm{CD} 30^{+}$, and $\mathrm{CD} 15^{-15}$

\section{EBV-ASSOCIATED T-CELL AND NATURAL KILLER CELL LPDS}

EBV is a ubiquitous herpes virus with tropism for $\mathrm{B}$ cells, but the infection of $\mathrm{T}$ cells and natural killer (NK) cells may lead to several EBV-related LPDs. EBV-positive T/NK LPD encompasses disease entities with a broad clinicopathologic spectrum (Table 2). ${ }^{16}$

Table 2. EBV-associated T-cell and NK cell lymphoproliferative diseases

\begin{tabular}{l}
\hline Disease \\
\hline EBV-associated hyperinflammatory syndrome \\
EBV-associated hemophagocytic lymphohistiocytosis \\
CAEBV-type T/NK cell disease \\
Systemic chronic active EBV infection of T cell or NK cell type \\
Cutaneous forms of CAEBV \\
Severe mosquito bite allergy \\
Hydroa vacciniforme-like lymphoproliferative disease \\
Malignant T/NK cell disease \\
Systemic EBV-positive T-cell lymphoma \\
Extranodal NKVT cell lymphoma, nasal type \\
Extranasal NK/T cell lymphoma \\
Aggressive NK cell leukemia \\
EBV-positive nodal NKTT cell lymphoma (provisional)
\end{tabular}

EBV, Epstein-Barr virus; CAEBV, chronic active EBV; NK, natural killer.

\section{EBV-associated hemophagocytic lymphohistiocytosis}

Hemophagocytic lymphohistiocytosis (HLH) is a clinicopathologic syndrome encompassing a markedly dysregulated immune response and hypercytokinemia. HLH is characterized clinically by fever, splenomegaly, and cytopenias, and histologically by hemophagocytosis. The supportive laboratory findings for HLH are as follows: extremely high serum level of ferritin, lactate dehydrogenase, soluble CD25, and elevated viral capsid. EBV associated $\mathrm{HLH}$ accounts for $40 \%$ of $\mathrm{HLH}$.

Histologically, EBV-positive T-cells and hemophagocytosing histiocytes are scattered in the sinusoids of the bone marrow and liver. The T-cells express CD8 and granzyme B. Even though it is uncommon, NK cells can be infiltrated in HLH. ${ }^{17}$

$\mathrm{HLH}$ can be effectively controlled in most patients (more than $90 \%$ ), but the other $10 \%$ often die of fulminant disease.

\section{CAEBV infection of T-cell or NK-cell types, systemic}

CAEBV infection was initially defined as follows: (1) markedly abnormal EBV antibody titer; (2) histologic evidence of organ involved- interstitial pneumonia, hypoplasia of the bone marrow, uveitis, lymphadenitis, persistent hepatitis, or splenomegaly; and (3) increased EBV RNA in affected tissue. ${ }^{18}$

Clinically, CAEBV-T/NK is a disease of children but is also detected in young adults and even in middle-aged older adults with a mean age of 11.3 years. ${ }^{19}$ The symptoms are usually prolonged fever, hepatomegaly, splenomegaly, thrombocytopenia, anemia, and lymphadenopathy. ${ }^{19}$ Life-threatening complications include hemophagocytic syndrome, interstitial pneumonia, malignant lymphoma, coronary aneurysms, and central nervous system involvement. All patients have elevated levels of EBV DNA in their
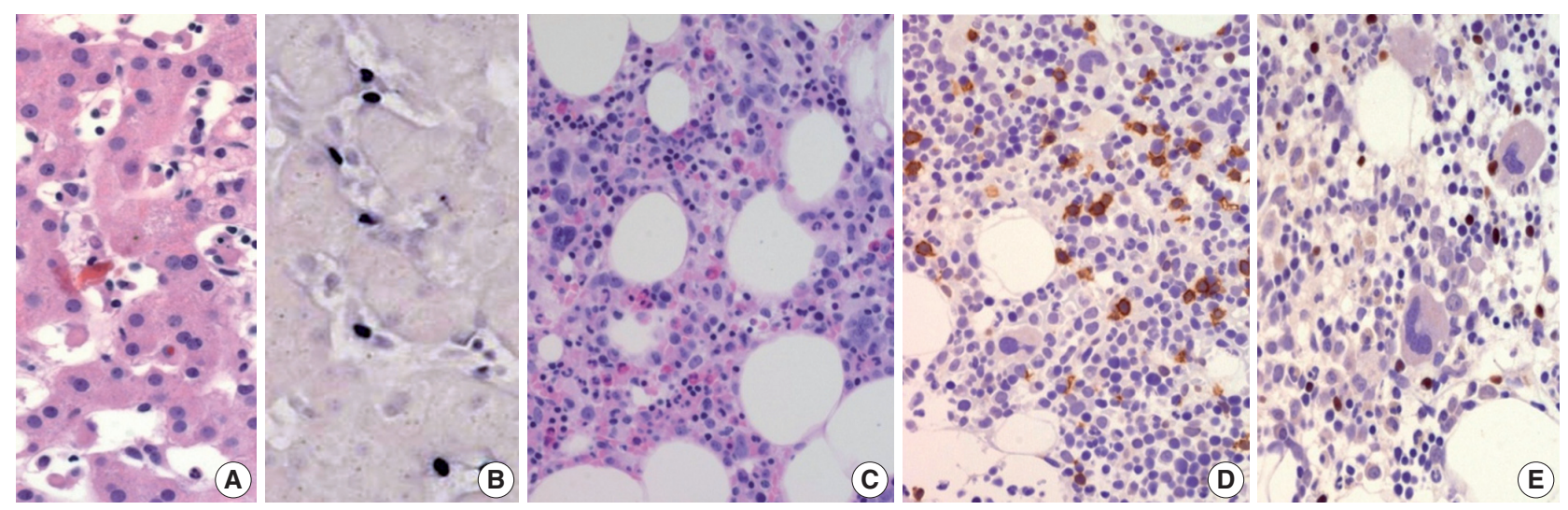

Fig. 2. Chronic active Epstein-Barr virus (EBV) infection of a T-cell or natural killer cell type, systemic (Courtesy of Dr. Y.H. Ko). (A) A 21-yearold man presented with severe oral ulcer, recurrent pneumonia, thrombocytopenia, and elevated liver enzymes for 2 years. Liver biopsy reveals atypical T lymphocytes infiltrating the sinusoidal and hepatic lobules. (B) EBV-encoded small RNA (EBER) in-situ hybridization exhibits positive signals in these T cells. (C) Bone marrow biopsy shows small lymphocytic infiltrate. (D) CD3 is expressed in most lymphocytes. (E) EBER in-situ hybridization also shows positive signals in T cells. 
blood, which is well-correlated with clinical severity. ${ }^{19}$

Morphologically, in patients with CAEBV-T/NK the lymph nodes exhibit paracortical hyperplasia with polymorphic and polyclonal lymphoid proliferation and large numbers of EBER positive cells. The liver exhibits portal or sinusoidal infiltration by small lymphocytes with no definite atypia (Fig. 2).

Ohshima et al. ${ }^{18}$ proposed a three-tier classification as follows: category A1 is polymorphic LPD with polyclonal proliferation of EBV-infected T cells or NK cells; category A2 is polymorphic LPD with monoclonal T/NK cells; and category $\mathrm{A} 3$ is monomorphic LPD of monoclonal T cells.

\section{Severe mosquito bite allergy}

A severe mosquito bite allergy is a cutaneous manifestation of chronic EBV infection characterized by intense local skin symptoms, such as erythema, bullae, ulcers, and scarring. The systemic symptoms such as fever, lymphadenopathy, and liver dysfunction are developed after mosquito bites, vaccination, or injection.

The epidermis at the mosquito bite site exhibits necrosis and ulceration. The dermis reveals edema and infiltration of polymorphonuclear leukocytes, nuclear debris, and extravasated erythrocytes with fibrinoid necrosis of small vessels. The infiltrating small lymphocytes extend from the dermis to the subcutis in an angiocentric pattern. EBV-positive cells represent 3\%-10\% of infiltrating lymphocytes. ${ }^{20}$

\section{Hydroa vacciniforme-like LPD}

Hydroa vacciniforme (HV)-like LPD is one of the cutaneous forms of CAEBV. It is initially described as an EBV positive polyclonal or monoclonal T/NK LPD, characterized by blistering photodermatoses in childhood and healed with vacciniform scarring. ${ }^{21}$ It is clinically divided into two types. The classic type is a self-limited disease with vesicles on sun-exposed areas in adolescence or young adulthood. Severe HV-type tends to exhibit more extensive skin lesions and systemic manifestations of fever, hepatomegaly, serologic abnormalities, and peripheral NK lymphocytosis. The severe type of $\mathrm{HV}$ often progresses to $\mathrm{EBV}$-associated NK/T-cell malignancy. ${ }^{22,23}$

Morphologic findings of $\mathrm{HV}$ are epidermal reticular degeneration to spongiotic vesiculation with perivascular and periappendiceal lymphocytic infiltration with no definite cytologic atypia. Severe HV and HV-like T-cell lymphomas mimic those of classic HV, but the dermal infiltrates are more extensive and deeper, composed of variably atypical lymphocytes.

The immunophenotype of the classic $\mathrm{HV}$ is $\mathrm{CD}^{+}$or $\mathrm{CD}^{+} \mathrm{T}$ cells, but the severe form/lymphoma exhibits predominantly
CD8 ${ }^{+}$CTLs. The majority are $\alpha \beta$ T cells. A few cases involve $\alpha \beta$ $\mathrm{T}$ cells and rarely NK cells.

\section{Systemic EBV-positive T-cell lymphoma}

Systemic EBV-positive T-cell lymphoma of childhood and young adulthood is a fulminant illness of EBV-infected T cells with clonal proliferation and cytotoxic phenotype. The clinical manifestation of this lesion is a rapid clinical progression with multiple organ failure, sepsis, and death. A hemophagocytic syndrome is nearly always associated. Systemic EBV-positive T-cell lymphoma arising in patients with a history of CAEBV-T/NK develops in a median time of 35 months. ${ }^{24}$

Hyperplasia of histiocytes and marked hemophagocytic syndrome are also noted with increased small T-cells in the bone marrow, spleen, and liver. The paracortical zone of the lymph node is expanded with the depletion of B-cell areas. The degree of cytologic atypia in EBV-positive lymphocytes is variable.

\section{Extranodal NK/T cell lymphoma, nasal type}

Extranodal NK/T cell lymphoma is a prototype of EBV-associated T-cell LPD, which is characterized by frequent necrosis, angiocentric growth, cytotoxic phenotype and a strong association with EBV. Since the nasal cavity is the most commonly involved site, the nasopharynx and upper aerodigestive areas including the nasal cavity disclose progressively destructive and ulcerative lesions or obstructive symptoms due to mass effects. ${ }^{25}$

\section{Extranasal NK/T cell lymphoma}

Extranasal NK/T cell lymphomas frequently involve the skin, gastrointestinal tract, testis, and soft tissue. Most patients present at a higher stage with multiple areas of involvement. ${ }^{26}$

The morphology of the involved sites is frequently ulcerated and necrotic. The cytologic composition varies ranging from small, medium, and large. Angiocentric growth accompanies diffuse necrosis, and vascular damage.

\section{Aggressive NK cell leukemia}

Aggressive NK-cell leukemia is a neoplasm of NK cells, which primarily involves peripheral blood and bone marrow. In contrast to conventional leukemia, the tumor cells may not be abundant in the peripheral blood and bone marrow. The average age of patients exhibiting this disorder is 39 years. The typical presentations include fever, hepatosplenomegaly, lymphadenopathy, and is complicated by hemophagocytic syndrome.

In histologic sections, there are diffuse, destructive and permeative infiltrates of monomorphic cells with a round to moderate 
rim of pale or amphophilic cytoplasm. Interspersed apoptotic bodies and zonal cell death are common. Angioinvasive and angiodestructive growth is also frequently noted. The clinical course is fatal.

\section{EBV-positive nodal NK/T cell lymphoma}

EBV-positive nodal T/NK cell lymphoma may involve a limited number of extranodal organs except for the nasal cavity, but the main bulk of the tumor is located in the lymph nodes. This entity is very rare with fewer than 100 cases being reported in the literature. ${ }^{27}$

The lymph nodes exhibit a diffuse infiltration of pleomorphic, variable sized cells. The cytomorphology of the tumor cells are more commonly centroblastoid, often anaplastic or plasmacytoid with some RS-like cells being noted. Some cases show extensive necrosis, many apoptotic bodies, and angiocentric growth patterns. The immunophenotype is as follows: $\mathrm{CD}^{+}, \mathrm{CD}^{+}$, $\mathrm{TIA}^{+}$, and granzyme $\mathrm{B}^{+} .^{28}$

The disease course is very aggressive, with a median survival of only 4 months.

\section{CONCLUSION}

Many new entities and concepts for EBV-positive LPDs have been added to the 2016 WHO classification, based on growing knowledge in the field of genetics and molecular virology. With this increased understanding of LPD the clinical and pathologic entities, we can perform EBV-ISH indicative of (1) clinically bordered between infection and neoplastic conditions; (2) past history of recurrent inappropriate immune response, especially in children/young adults or old age; and (3) a pathologically polymorphous inflammatory background (but not Hodgkin lymphoma). It helps that the final destination is the achievement of appropriate diagnosis and management of LPDs. Some entities are provisional and must wait for confirmation of additional data.

\section{Conflicts of Interest}

No potential conflict of interest relevant to this article was reported.

\section{REFERENCES}

1. Cohen JI. Epstein-Barr virus infection. N Engl J Med 2000; 343: 48192.
2. Pittaluga S, Said J. Virally associated B cell lymphoproliferative disease. In: Jaffe E, Arbor DA, Campo E, Harris NL, QuintanillaFend L, eds. Hematopathology. 2nd ed. Philadelphia: Elsevier Heealth Science; 2016; 547-607.

3. Swerdlow SH, Campo E, Pileri SA, et al. The 2016 revision of the World Health Organization classification of lymphoid neoplasms. Blood 2016; 127: 2375-90.

4. Chaganti S, Heath EM, Bergler W, et al. Epstein-Barr virus colonization of tonsillar and peripheral blood B-cell subsets in primary infection and persistence. Blood 2009; 113: 6372-81.

5. Vouloumanou EK, Rafailidis PI, Falagas ME. Current diagnosis and management of infectious mononucleosis. Curr Opin Hematol 2012; 19: 14-20.

6. Fletcher C. Diagnostic histopathology of tumors. 3rd ed. Philadelphia: Churchill Livingstone/Elsevier, 2007; 1260-1.

7. Lekstrom-Himes JA, Dale JK, Kingma DW, Diaz PS, Jaffe ES, Straus SE. Periodic illness associated with Epstein-Barr virus infection. Clin Infect Dis 1996; 22: 22-7.

8. Cho EY, Kim KH, Kim WS, Yoo KH, Koo HH, Ko YH. The spectrum of Epstein-Barr virus-associated lymphoproliferative disease in Korea: incidence of disease entities by age groups. J Korean Med Sci 2008; 23: $185-92$

9. Cohen JI, Jaffe ES, Dale JK, et al. Characterization and treatment of chronic active Epstein-Barr virus disease: a 28-year experience in the United States. Blood 2011; 117: 5835-49.

10. Swerdlow SH, Campo E, Harris NL, et al. WHO classification of tumors of hematopoietic and lymphoid tissues. 4th ed. Lyon: IARC Press, 2008.

11. Nicolae A, Pittaluga S, Abdullah S, et al. EBV-positive large B-cell lymphomas in young patients: a nodal lymphoma with evidence for a tolerogenic immune environment. Blood 2015; 126: 863-72.

12. Dojcinov SD, Venkataraman G, Raffeld M, Pittaluga S, Jaffe ES. EBV positive mucocutaneous ulcer--a study of 26 cases associated with various sources of immunosuppression. Am J Surg Pathol 2010; 34: 405-17.

13. Hongyo T, Kurooka M, Taniguchi E, et al. Frequent p53 mutations at dipyrimidine sites in patients with pyothorax-associated lymphoma. Cancer Res 1998; 58: 1105-7.

14. Katzenstein AL, Carrington CB, Liebow AA. Lymphomatoid granulomatosis: a clinicopathologic study of 152 cases. Cancer 1979; 43: 360-73.

15. Song JY, Pittaluga S, Dunleavy K, et al. Lymphomatoid granulomatosis: a single institute experience: pathologic findings and clinical correlations. Am J Surg Pathol 2015; 39: 141-56.

16. Kimura H, Ito Y, Kawabe S, et al. EBV-associated T/NK-cell lymphoproliferative diseases in nonimmunocompromised hosts: prospec- 
tive analysis of 108 cases. Blood 2012; 119: 673-86.

17. Toga A, Wada T, Sakakibara Y, et al. Clinical significance of cloned expansion and CD5 down-regulation in Epstein-Barr Virus (EBV)infected CD8+ T lymphocytes in EBV-associated hemophagocytic lymphohistiocytosis. J Infect Dis 2010; 201: 1923-32.

18. Ohshima K, Kimura H, Yoshino T, et al. Proposed categorization of pathological states of EBV-associated T/natural killer-cell lymphoproliferative disorder (LPD) in children and young adults: overlap with chronic active EBV infection and infantile fulminant EBV TLPD. Pathol Int 2008; 58: 209-17.

19. Ko YH, Kim HJ, Oh YH, et al. EBV-associated T and NK cell lymphoproliferative disorders: consensus report of the 4th Asian Hematopathology Workshop. J Hematopathol 2012; 5: 319-24.

20. Cohen JI, Kimura H, Nakamura S, Ko YH, Jaffe ES. Epstein-Barr virusassociated lymphoproliferative disease in non-immunocompromised hosts: a status report and summary of an international meeting, 8-9 September 2008. Ann Oncol 2009; 20: 1472-82.

21. Park S, Ko YH. Epstein-Barr virus-associated T/natural killer-cell lymphoproliferative disorders. J Dermatol 2014; 41: 29-39.

22. Miyake T, Yamamoto T, Hirai Y, et al. Survival rates and prognostic factors of Epstein-Barr virus-associated hydroa vacciniforme and hypersensitivity to mosquito bites. Br J Dermatol 2015; 172: 56-63.
23. Cho KH, Kim CW, Heo DS, et al. Epstein-Barr virus-associated peripheral T-cell lymphoma in adults with hydroa vacciniforme-like lesions. Clin Exp Dermatol 2001; 26: 242-7.

24. Quintanilla-Martinez L, Kumar S, Fend F, et al. Fulminant EBV(+) T-cell lymphoproliferative disorder following acute/chronic EBV infection: a distinct clinicopathologic syndrome. Blood 2000; 96: 443-51.

25. Hong M, Lee T, Kang SY, Kim SJ, Kim W, Ko YH. Nasal-type NK/ $\mathrm{T}$-cell lymphomas are more frequently $\mathrm{T}$ rather than NK lineage based on T-cell receptor gene, RNA, and protein studies: lineage does not predict clinical behavior. Mod Pathol 2016; 29: 430-43.

26. Chan JK, Sin VC, Wong KF, et al. Nonnasal lymphoma expressing the natural killer cell marker CD56: a clinicopathologic study of 49 cases of an uncommon aggressive neoplasm. Blood 1997; 89: 450113.

27. Kato S, Asano N, Miyata-Takata T, et al. T-cell receptor (TCR) phenotype of nodal Epstein-Barr virus (EBV)-positive cytotoxic T-cell lymphoma (CTL): a clinicopathologic study of 39 cases. Am J Surg Pathol 2015; 39: 462-71.

28. Jeon YK, Kim JH, Sung JY, Han JH, Ko YH; Hematopathology Study Group of the Korean Society of Pathologists. Epstein-Barr virus-positive nodal T/NK-cell lymphoma: an analysis of 15 cases with distinct clinicopathological features. Hum Pathol 2015; 46: 981-90. 